\title{
Interfacial phase transitions in a far-from-equilibrium magnetic growth model
}

\author{
Julián Candia ${ }^{1}$ and Ezequiel V Albano \\ Instituto de Investigaciones Fisicoquímicas Teóricas y Aplicadas (INIFTA), CONICET, UNLP, \\ Sucursal 4, Casilla de Correo 16, (1900) La Plata, Argentina \\ E-mail: ealbano@inifta.unlp.edu.ar
}

Received 11 December 2001, in final form 25 February 2002

Published 2 May 2002

Online at stacks.iop.org/JPhysCM/14/4927

\begin{abstract}
The irreversible growth of a magnetic film with spins having two possible orientations is studied in three-dimensional confined geometries of size $L \times L \times M$, where $M \gg L$ is the growing direction. A competing situation with two opposite short-range surface magnetic fields $H$ of the same magnitude is analysed. Due to the antisymmetric condition considered, an interface between domains with spins having opposite orientations develops along the growing direction. Such an interface undergoes a localization-delocalization transition that is the precursor of a wetting transition in the thermodynamic limit, in qualitative agreement with observations made under equilibrium conditions. However, in contrast to its equilibrium counterparts, the film also exhibits a growing interface that undergoes a concave-convex transition in the growth mode. The phase diagram on the $H$ versus $T$ plane is first obtained for a finite system, and exhibits eight different regions. Subsequently, the phase diagram corresponding to the thermodynamic limit is obtained by extrapolation. It is shown that in the latter only six regions remain. The relevant physical properties of all of these regions are discussed in detail.
\end{abstract}

\section{Introduction}

The interaction of a saturated gas in contact with a wall or a substrate may result in the occurrence of very interesting wetting phenomena, where a macroscopically thick liquid layer condenses at the wall, while the bulk fluid may remain in the gaseous phase [1-5]. The wetting of solid surfaces by a fluid is a phenomenon of primary importance in many fields of practical technological applications (lubrication, efficiency of detergents, oil recovery in porous material, stability of paint coatings, interaction of macromolecules with interfaces, etc [1]).

1 Present and permanent address: Departamento de Física, Facultad de Ciencias Exactas, Universidad Nacional de La Plata, CC67, (1900) La Plata, Argentina. 
Surface enrichment and wetting layers have been observed experimentally in a great variety of systems, such as polymer mixtures [6-8] and adsorption of simple gases on alkali metal surfaces [9-11], with the recent addition of $\mathrm{Hg}$ to the adsorption species exhibiting this kind of transition phenomenon [12,13], and also hydrocarbons on mica [14], etc.

The study of wetting transitions at interfaces has also attracted considerable theoretical interest [3-5], involving, among others, different approaches such as the mean-field GinzburgLandau method $[15,16]$, transfer matrix and Pfaffian techniques $[17,18]$, density matrix renormalization group methods [19], solving the Cahn-Hilliard equation [20], using molecular dynamics simulations [21], solving self-consistent field equations [22], and by means of extensive Monte Carlo simulations [23-27].

So far, the considerable progress due to all these studies of wetting transitions has been achieved for systems under equilibrium conditions. In contrast, the study of wetting phenomena under non-equilibrium conditions has received much less attention. For instance, Hinrichsen et al [28] have recently introduced a non-equilibrium growth model of a onedimensional interface interacting with a substrate. The interface evolves via adsorptiondesorption processes, which depart from detailed balance. Then, on changing the relative rates of these processes a transition from a binding to a nonbinding phase has been reported [28].

Within this context, the aim of this work is to perform an extensive numerical study of the irreversible growth of a magnetic material confined between parallel walls where competing surface magnetic fields act. For this purpose, a variant of the irreversible Eden growth model [29], in which particles are replaced by spins that can adopt two different orientations, is investigated. It is shown that the interplay between confinement and growth mode leads to a physically rich phase diagram. It should also be remarked that, although the discussion is presented here in terms of a magnetic language, the relevant physical concepts can rather straightforwardly be extended to other systems such as fluids, polymers, and binary mixtures. Apart from the fundamental interest in understanding this complex physical situation, it may well play a key role in the development of technologies such as micromagnetic materials, microfluidics, self-assembly of three-dimensional structures, adhesives, lubricants, and coatings. Indeed, wetting phenomena under far-from-equilibrium conditions are expected to be of wide application to describe a great variety of processes actually encountered in practice.

Furthermore, the proposed study establishes a link with recent investigations of irreversible growth processes. In fact, the study of growth systems under far-from-equilibrium conditions is a subject that has attracted great attention during the last few decades. Nowadays, this interdisciplinary field has shown a rapid progress due to both its interest in many subfields of physics, chemistry, and biology, and its relevance in numerous technological applications. Recent progress in our understanding of growth phenomena, with special emphasis on the properties of rough interfaces, has been extensively reviewed [30-34].

Also, the study of wetting phenomena in far-from-equilibrium systems under confinement has an extra ingredient of theoretical interest due to the delicate interplay between surface and bulk properties. Indeed, from the experience gained studying equilibrium systems, it is well known that, using confined geometries with restricted dimensionality, the effects of statistical fluctuations are more pronounced [23-27,35-41], leading to a new and rich physical behaviour, which eventually may be the precursor of the actual critical behaviour only observed in the thermodynamic limit. Within this context, in the present work it is shown that in far-fromequilibrium systems, the subtle interplay between finite-size effects, wetting, and interface growth mechanisms leads to more rich and complex physical features than in the equilibrium counterpart. In fact, a complex phase diagram that exhibits a localization-delocalization transition in the interface that runs along the walls and a change of the curvature of the growing interface running perpendicularly to the walls is evaluated and discussed, first for finite-size 
systems, and subsequently for the extrapolated infinite system.

This paper is organized as follows: in section 2 we give details on the simulation method, section 3 is devoted to the presentation and discussion of the results, while the conclusions are finally stated in section 4 .

\section{The model and the simulation method}

In the classical Eden model [29] on the square lattice, the growth process starts by adding particles to the immediate neighbourhood (the perimeter) of a seed particle. Subsequently, particles are stuck at random to perimeter sites. This growth process leads to the formation of compact clusters with a self-affine interface [31-34]. The magnetic Eden model (MEM) [42] considers an additional degree of freedom due to the spin of the growing particles. Early studies of the MEM have been performed using a single seed placed at the centre of the sample [42], but some subsequent investigations [43-46] have adopted instead $(d+1)$-dimensional rectangular geometries. Following the latter approach, in the present work the MEM in $(2+1)$ dimensions is studied using a rectangular geometry, $L \times L \times M$ (with $M \gg L$ ). Figure 1 illustrates the general set-up assumed. The location of each site on the lattice is specified through its rectangular coordinates $(i, j, k)(1 \leqslant i, j \leqslant L, 1 \leqslant k \leqslant M)$. The starting seed for the growing cluster is a plane of $L \times L$ parallel-oriented spins placed at $k=1$, and cluster growth takes place along the positive longitudinal direction (i.e., $k \geqslant 2$ ). Periodic boundary conditions are chosen along one of the transverse directions (say in the $i$-direction), while open boundary conditions are adopted along the remaining transverse direction. Competing surface magnetic fields $H>0\left(H^{\prime}=-H\right)$ acting on the sites placed at $j=1(j=L)$ are considered. Then, assuming that each spin $S_{i j k}$ may adopt two possible orientations, namely up and down (i.e. $S_{i j k}= \pm 1$ ), clusters are grown by selectively adding spins to perimeter sites, which are defined as the nearest-neighbour (NN) empty sites of the already occupied ones. Considering a ferromagnetic interaction of strength $J>0$ between NN spins, the energy $E$ of a given configuration of spins is given by

$$
E=-\frac{J}{2}\left(\sum_{\left\langle i j k, i^{\prime} j^{\prime} k^{\prime}\right\rangle} S_{i j k} S_{i^{\prime} j^{\prime} k^{\prime}}\right)-H\left(\sum_{\left\langle i k, \Sigma_{1}\right\rangle} S_{i 1 k}-\sum_{\left\langle i k, \Sigma_{L}\right\rangle} S_{i L k}\right),
$$

where $\left\langle i j k, i^{\prime} j^{\prime} k^{\prime}\right\rangle$ means that the summation in the first term is taken over all occupied NN sites, while $\left\langle i k, \Sigma_{1}\right\rangle,\left\langle i k, \Sigma_{L}\right\rangle$ denote summations carried over occupied sites on the surfaces $\Sigma_{1}, \Sigma_{L}$ (defined as the $j=1$ and $L$ planes, respectively). Thus, setting the Boltzmann constant equal to unity $\left(k_{B} \equiv 1\right)$ and measuring absolute temperature, energy, and magnetic fields in units of $J$, the change of energy $\Delta E$ involved in the addition of a spin $S_{i j k}$ to the system is given by

$$
\Delta E=-S_{i j k}\left(\sum_{\left\langle i j k, i^{\prime} j^{\prime} k^{\prime}\right\rangle} S_{i^{\prime} j^{\prime} k^{\prime}}+H\left(\delta_{j 1}-\delta_{j L}\right)\right),
$$

where the summation $\left\langle i j k, i^{\prime} j^{\prime} k^{\prime}\right\rangle$ is taken over occupied NN sites keeping $i, j, k$ fixed, and $\delta_{j 1}, \delta_{j L}$ are standard Kronecker delta symbols. Therefore, the probability for a perimeter site to be occupied by a spin $S_{i j k}$ is proportional to the Boltzmann factor $\exp (-\Delta E / T)$, where $\Delta E$ is given by equation (2). At each step, the probabilities of adding up and down spins to a given site have to be evaluated for all perimeter sites. After proper normalization of the probabilities, the growing site and the orientation of the spin are determined through standard Monte Carlo techniques. Although both the interaction energy and the Boltzmann probability distribution considered for the MEM are similar to those used for the Ising model with surface magnetic 


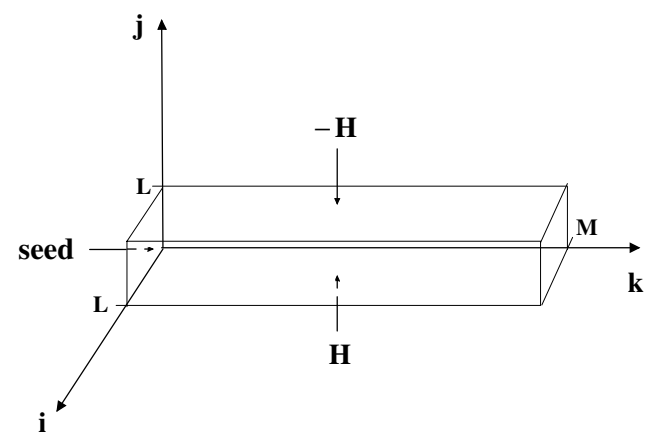

Figure 1. The general set-up for the MEM in a $(2+1)$-dimensional rectangular geometry. The system grows along the positive longitudinal direction from a seed constituted by $L \times L$ paralleloriented spins placed at $k=1$, as indicated. Competing surface magnetic fields are applied on the surfaces $j=1$ and $L$, while periodic boundary conditions are assumed along the $i$-direction. The slices shown in figure 4, obtained for different temperatures and magnetic fields, illustrate typical growth regimes.

fields [23], it must be stressed that these two models operate under extremely different conditions, namely the MEM describes the irreversible growth of a magnetic material and the Ising model is suitable for the study of a magnetic system under equilibrium conditions. In the MEM, the position and orientation of all deposited spins remain fixed. The non-equilibrium nature of the MEM is clear from the fact that the extensive thermodynamic variables (such as energy, entropy, and volume) grow monotonically with time and tend to diverge. Furthermore, during the growth process, the system develops a rough growth interface and evolves mainly along the longitudinal direction $k$ (see figure 1). However, some lattice sites could remain empty even well within the system's bulk. Since at each growth step all perimeter sites are potential candidates for being occupied by the next spin to be added, these holes become gradually filled. It may appear that the $k$-coordinate is something like the 'time' in a kinetic Ising model. However, this is not strictly true because already deposited spins at position $k$ effectively affect spin growth at position $k^{\prime}<k$, and this would mean causality violation.

Far behind the active growth interface, the system is compact and frozen. When the growing cluster interface is close to reaching the limit of the sample $(k=M)$ one can compute the relevant properties of the irreversibly frozen cluster's bulk (in the region where the growing process has definitively stopped), thereafter erase the useless frozen bulk, and finally shift the growing interface towards the lowest possible coordinate $k$. Hence, repeatedly applying this procedure, the growth process is not limited by the lattice length $M$.

It should be noticed that this paper involves a large computational effort. On the one hand, as will be seen below, the observables of interest (e.g. the susceptibility) are averaged over many transverse planes of size $L \times L$. In order to obtain acceptably small statistical errors, averages over $\sim 10^{5-6}$ planes in the stationary regime are typically required. So, in the present work clusters having up to $\sim 10^{9}$ spins have been grown. On the other hand, the update algorithm is quite slow as compared with standard Ising simulations, since the growing probability has to be computed after each deposition event.

\section{Results and discussion}

Recent investigations [44] have shown that the magnetic Eden growth process in a stripped $(d+1)$-dimensional geometry (with $d=1,2)$ is characterized by an initial transient of average 
length $l_{T r}$, followed by a non-equilibrium stationary state that is independent of the starting seed. It has also been shown that the MEM in $d=1$ dimension is non-critical (i.e., it only exhibits an ordered phase at $T=0$ ), while the MEM in $d=2$ dimensions undergoes an orderdisorder thermal transition of second order at the temperature $T_{c}=0.69 \pm 0.01$. Moreover, the critical exponents associated with the continuous phase transition have been found to agree, within error bars, with those of the Ising model in two dimensions. Hence, the reported findings have led to the conjecture that the $(d+1)$-dimensional MEM and the $d$-dimensional Ising model behave identically (except as regards finite-size differences that vanish in the thermodynamic limit) at criticality for all $d$ [44].

At this stage it is appropriate to briefly recall that a confined Ising film with competing surface fields undergoes an equilibrium wetting transition. Indeed, when an Ising film is confined between two competing walls a distance $L$ apart from each other, so that the surface magnetic fields $(H)$ are of the same magnitude but opposite direction, it is found that the competing fields cause the emergence of an interface that undergoes a localization-delocalization transition. This transition shows up at an $L$-dependent temperature $T_{w}(L, H)$ that is the precursor of the true wetting transition temperature $T_{w}(H)$ of the infinite system $[16,23,24]$.

In view of the non-trivial correspondence established between the $(d+1)$-dimensional MEM and the $d$-dimensional Ising model, we should also expect an Ising-like wetting transition for the MEM. In fact, on applying surface magnetic fields of opposite signs to the MEM, we should expect to obtain a well defined phase transition curve between wet and non-wet states on the $H-T$ plane. In order to deal with a phase transition that will remain in the thermodynamic limit $(L \rightarrow \infty)$, one should devote attention to the $(d+1)$-dimensional MEM with $d \geqslant 2$ since, as already pointed out, the MEM is non-critical for $d=1$. For this purpose, we have studied the $(2+1)$-dimensional MEM with magnetic fields $H$ and $H^{\prime}=-H$ applied to the surfaces $\Sigma_{1}$ and $\Sigma_{L}$, respectively (recall section 2). As in previous investigations [44], the mean transverse magnetization is defined as

$$
m(k, L, T, H)=\frac{1}{L^{2}} \sum_{i, j=1}^{L} S_{i j k}
$$

for $k>l_{T r}$, in order to exclude the initial transient. Furthermore, it is assumed that the finitesize ( $L$-dependent) susceptibility can be defined in terms of order parameter fluctuations in the same manner as for equilibrium systems, namely

$$
\chi=\frac{L^{2}}{T}\left(\left\langle m^{2}\right\rangle-\langle|m|\rangle^{2}\right),
$$

where $\langle\cdots\rangle$ means the average taken over a sufficiently large number of transverse planes in the stationary regime. Then, using a standard procedure [23], the localization-delocalization transition curve (on the $H-T$ plane) corresponding to the up-down interface running along the walls can be obtained considering that a point with coordinates $\left(H_{w}, T_{w}\right)$ on this curve maximizes $\chi(H, T)$. Figure 2 shows plots of $\chi$ versus $T$ for several values of $H$ and the fixed lattice size $L=12$, illustrating the method used to trace the size-dependent localizationdelocalization transition curve, which is shown in figure 3 (open squares). As in the case of the Ising model, this quasi-wetting transition refers to a transition between a non-wet state that corresponds to a localized interface bound to one of the confinement walls, and a wet state associated with a delocalized domain interface centred between roughly equal domains of up and down spins. The localization-delocalization transition in a confined system is indeed the precursor of the true wetting transition that occurs in the thermodynamic limit $[16,23,24]$. In fact, there is observed a finite jump in the wetting layer thickness that takes place as a result of 


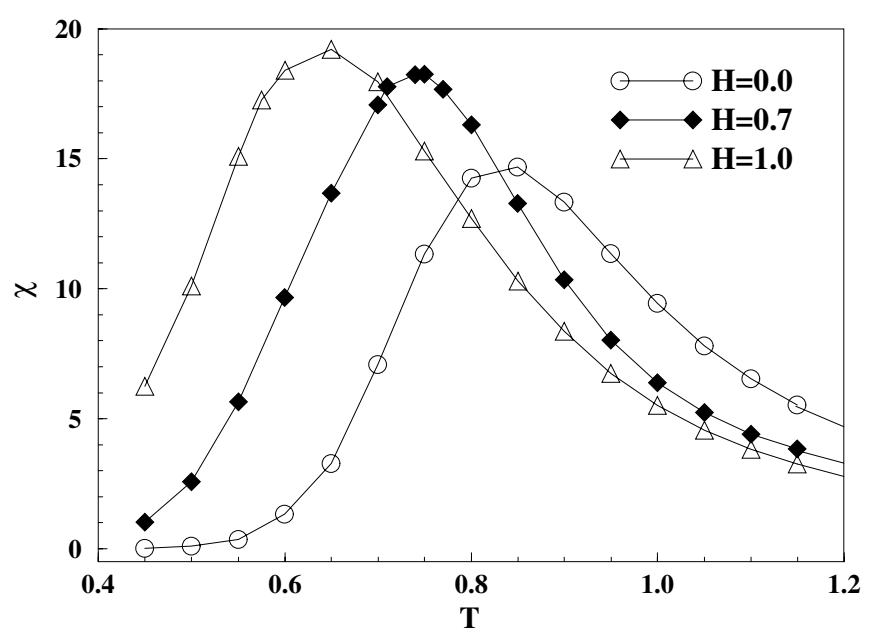

Figure 2. Plots of $\chi$ versus $T$ for a fixed lattice size $L=12$ and several values of $H$, as indicated. If $T_{w}$ is the temperature that corresponds to the maximum of $\chi$ for a given fixed value of $H=H_{w}$, then $\left(H_{w}, T_{w}\right)$ is a point on the wet-non-wet transition curve, as follows from standard procedures [23].

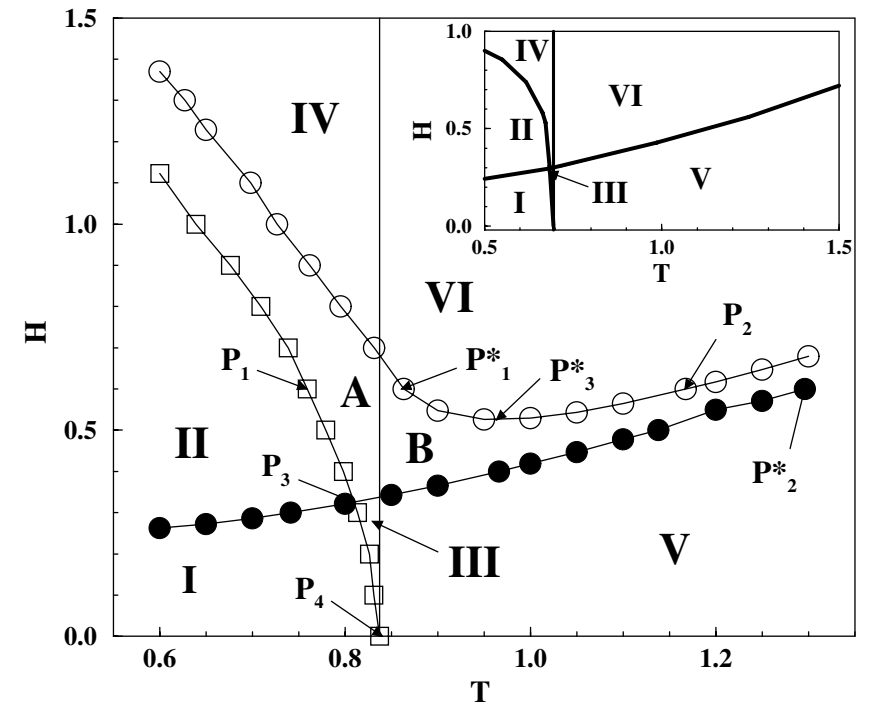

Figure 3. The $H-T$ phase diagram corresponding to a lattice of size $L=12$. The vertical straight line at $T_{c}(L)=0.84$ corresponds to the $L$-dependent critical temperature, which separates the low-temperature ordered phase from the high-temperature disordered phase. Open (filled) circles refer to the transition between non-defined and concave (convex) growth regimes, and squares stand for the Ising-like localization-delocalization transition curve. Eight different regions are distinguished, as indicated in the figure. Also indicated are seven representative points that are discussed in the text. The inset shows the phase diagram corresponding to the thermodynamic limit composed of six different regions.

the finite size of the system. As the lattice size is increased, the magnitude of the jump grows and diverges in the $L \rightarrow \infty$ limit, as expected for a continuous wetting transition.

Let us now discuss the critical temperature associated with the bulk order-disorder phase transition. As is well known from finite-size scaling theory, there is some degree of arbitrariness 
in locating the $L$-dependent critical temperature $T_{c}(L)$ of a finite system. However, the critical point $T_{c}$ of the infinite system, obtained by extrapolating $T_{c}(L)$ to the $L \rightarrow \infty$ limit, is unique and independent of any particular choice for the finite-size critical point. Let us first consider the case with $H=0$, defining the $L$-dependent critical temperature as given by the peak of the susceptibility at zero surface field, to ensure consistency with the evaluation of the localization-delocalization quasi-wetting transition. Indeed, under this assumption, the quasi-wetting curve $T_{w}(L, H)$ and the critical point $T_{c}(L)$ coincide by definition at $H=0$. For $L=12$, the critical point so defined is $T_{c}(L=12)=0.84$, and is shown in figure 2 by a vertical straight line. Using larger and larger lattices, correspondingly smaller finite-size critical points are found, which tend to the actual critical point of the $(2+1)$-dimensional MEM, namely $T_{c}=0.69 \pm 0.01$ [44]. Before exploring the case $H>0$, it is appropriate to recall a long-standing discussion on this topic generated in the field of equilibrium critical phenomena. Parry and Evans $[15,47]$ claim that the critical temperature for a finite system depends on the surface magnetic fields, and only differs from the wetting temperature of the infinite system $\left(T_{w}(H)\right)$ by a term that vanishes in the thermodynamic limit. Indeed, they suggest a scaling ansatz such that $T_{w}(H)-T_{c}(L, H)$ is of order $L^{-1 / \beta_{s}}$, where $\beta_{s}$ is the exponent that describes the growth of the wetting layer. However, Swift et al [16] and Indekeu et al [48] propose that $T_{c}(L, H)$ is actually the temperature of a shifted wetting transition (hence called the quasiwetting transition temperature $T_{w}(L, H)$ ), which is different from the bulk critical point $T_{c}(L)$, and such that $T_{w}(L, H)$ tends for $L \rightarrow \infty$ to the actual wetting temperature $T_{w}(H)$. In the case of the MEM, general considerations, supported by our numerical results, appear to favour the latter proposal, so the bulk critical point is independent of $H$ and hence clearly different from the quasi-wetting temperature. However, it should be remarked that the controversy has been established for systems under equilibrium and the present study of the MEM corresponds to far-from-equilibrium conditions.

Let us first consider an increase in the surface fields from $H=0$ for a system within the ordered phase (i.e., for $T<T_{c}(L)$ ). Since fluctuations in the bulk are governed only by the temperature, it clearly turns out that the bulk will remain in its ordered state irrespective of the applied field. Indeed, as will be discussed below in detail, an increase in the fields favours the formation of a stable longitudinal interface between domains of up and down spins. However, within each domain, it is clear that the state of order will depend only in fluctuations driven by the temperature. Hence, surface fields applied to an ordered system below $T_{c}(L)$ will eventually favour the coexistence of oppositely oriented ordered domains, but are not capable of generating disorder within each domain. These arguments are strongly supported by our simulations. For instance, figures $4(a)-(c)$ show typical snapshot configurations that correspond to nearly the same temperature (below $T_{c}(L=32)=0.76$ ) and several different magnetic fields. As expected from our considerations, the fields appear to support the formation of the longitudinal interface between opposite spin domains, but do not affect the bulk ordered state within each domain. In particular, it should be noticed that figure $4(c)$ corresponds to a field well above the corresponding one on the quasi-wetting curve (note that the snapshots correspond to $L=32$, and the associated transition curves are shifted to the left with respect to those for $L=12$, shown in the phase diagram of figure 3$)$. If $T_{w}(L, H)$ were to be the system's critical point, we should expect a system beyond the quasi-wetting curve to be disordered, in remarkable contrast to the ordered configuration shown by figure 4(c). Moreover, as a check of consistency, one can compare the configurations shown in figures $4(c)$ and $(e)$, that correspond to nearly the same fields and differ in temperature. It is thus evident that the bulk's orderdisorder phase transition occurs at a temperature far away from the quasi-wetting transition curve, and consistent with $T_{c}(L=32)=0.76$.

Further insights into the role of $H$ acting within the bulk ordered phase $T<T_{c}(L)$ can 




Figure 4. Snapshot pictures showing a longitudinal slice given by a fixed value of the transverse coordinate $i$. Grey (black) points correspond to up (down) spins. The surface field on the upper (lower) confinement wall is positive (negative). The snapshots correspond to a lattice size $L=32$ and several different values of temperature and surface fields: (a) $H=0.05, T=0.6 ;(b) H=0.5$, $T=0.55 ;(c) H=1.4, T=0.6$; (d) $H=0.1, T=1.0 ;(e) H=1.6, T=1.4$; and $(f) H=0.20$, $T=0.82$.

be gained by means of the following procedure. Let us focus our attention on the stationary regime considering all completely filled columns directed along the $j$-direction, which are formed by $L$ spins and are identified through the values of the remaining coordinates $i$ and $k$. For any given column $(i, k)$, a bond to each pair of NN sites occupied by oppositely oriented spins is assigned. Summing over the whole column, $n_{b}(i, k)$ is defined as the total number of bonds for that column, so $n_{b}=0$ for parallel-oriented spins and $n_{b}=L-1$ for alternating up-down NN spins. Since all columns are statistically independent, the system is allowed to grow for a sufficiently long time and averages are taken over all filled columns. In this way, the normalized bond probability distribution $P\left(n_{b}\right)$ can be computed as a function of temperature, surface magnetic fields, and lattice size. For the purposes of the present discussion, it suffices to fix $L=12$ and consider the effects of increasing the fields for a given value of temperature below $T_{c}(L=12)=0.84$. Figure 5 shows the bond probability distribution $P\left(n_{b}\right)$ versus $n_{b}$ for $T=0.6$ and several values of $H$. It is observed that $P\left(n_{b}\right) \approx 0$ for $n_{b} \geqslant 2$, irrespective of the field. Hence, it is concluded that the system remains in its ordered state independently of $H$, and that the role of the magnetic field is that of driving the system from a state constituted by a single domain $\left(P\left(n_{b}=0\right) \approx 1\right)$ to a state formed by two oppositely oriented ordered domains $\left(P\left(n_{b}=1\right) \approx 1\right)$. Figure 6 shows the mean number of bonds per column $\left\langle n_{b}\right\rangle$ as a function of $H$, for three different temperatures. As expected, in all cases the field drives the crossover from a single ordered domain to two opposite ordered domains.

So, our discussion concerning the location of the critical temperature associated with the bulk order-disorder phase transition can be summarized by stating that we find compelling evidence for interpreting $T_{w}(L, H)$ as a quasi-wetting transition temperature, clearly different from the finite-size critical temperature $T_{c}(L)$. Furthermore, our results are consistent with the assumption of a field-independent critical point $T_{c}(L)$, since the magnetic field appears to play no role in the state of order within each magnetic domain.

Since the MEM is a non-equilibrium kinetic growth model, it also allows the identification of another kind of phase transition, namely a morphological transition associated with the curvature of the growing interface of the system [43]. To avoid confusion, we remark that the term growing interface is used here for the transverse interface between occupied and empty 




Figure 5. Plots of the normalized bond probability distribution $P\left(n_{b}\right)$ versus $n_{b}$ for $L=12$, $T=0.6$, and several values of $H$, as indicated. $P\left(n_{b}\right)$ is negligible for $n_{b}>3$, and thus it is not shown in the figure. The role of the magnetic field appears to be that of driving the system from a state constituted by a single domain $\left(P\left(n_{b}=0\right) \approx 1\right)$ to a state formed by two oppositely oriented ordered domains $\left(P\left(n_{b}=1\right) \approx 1\right)$.

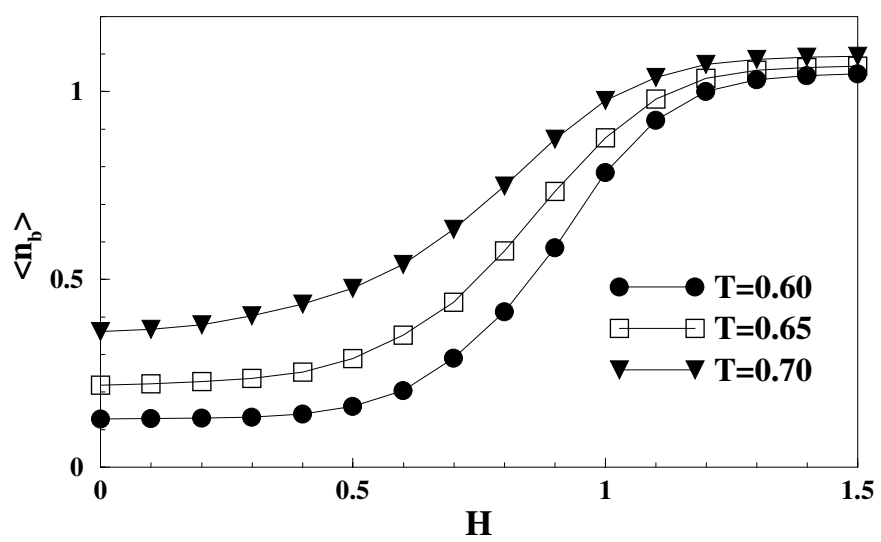

Figure 6. Plots of $\left\langle n_{b}\right\rangle$ versus $H$ for a fixed lattice size $L=12$ and several values of $T$, as indicated. As expected, in all cases the field drives the crossover from a single ordered domain to two opposite ordered domains.

lattice sites, while it was used above for the longitudinal interface between up- and down-spin domains.

Firstly, let us consider a longitudinal slice with a fixed value of $i$ in the range $1 \leqslant i \leqslant L$. In order to define the location of the growing interface at time $t$, it is assumed that each row contributes to the growing interface with the outermost perimeter site (i.e., the site with the largest value of the longitudinal coordinate $k$, for a given row number $j$ ) and that number is called $I_{j}(t)$. Then, the growing interface centre of mass, that is taken as the location of the growing interface at time $t, I(t)$, is given by

$$
I(t)=\frac{1}{L} \sum_{j=1}^{L} I_{j}(t) .
$$

Subsequently, one can evaluate the coordinates of the growing interface relative to its centre- 
of-mass location at time $t$, namely $I R_{j}(t) \equiv I_{j}(t)-I(t)$, for $j=1,2, \ldots, L$. In this way, it is possible to describe the growing interface at any time $t$ during the growing process just by evaluating the set $\left\{I R_{j}(t)\right\}$. However, one should be cautious at this point. In fact, since the applied surface fields are of equal intensity but have opposite direction, it turns out that the probability of occurrence of a given growing interface $\left\{I R_{j}\right\}$ must equal that corresponding to $\left\{I R_{j^{\prime}}\right\}$, where $j^{\prime}=L+1-j$. But then, unless the growth profile happens to be symmetric (i.e., invariant under $j \rightarrow L+1-j$, for all $j)$, the time average of equally probable growing interfaces $\left\{I R_{j}\right\},\left\{I R_{j^{\prime}}\right\}$ will lead to an unphysical symmetrized profile that is not representative of the actual shape of the growing interface. To avoid this problem, the following procedure is used. First, the largest value of the longitudinal coordinate $k$ that corresponds to a completely filled column is located. Then, by means of the sign of the total magnetization of that column, i.e. $S \equiv \operatorname{sgn}\left(\sum_{j} S_{i j k}\right)$, the orientation of the dominant spin domain in the active growing interface is identified. Supposing that, following the recipe given above, a given profile $\left\{I R_{j}(t)\right\}$ is obtained, then the growing interface location is redefined as $\left\{I R_{j^{*}}(t)\right\}$, where $j^{*} \equiv j$ if $S=+1$ and $j^{*} \equiv j^{\prime}=L+1-j$ if $S=-1$, for all $j$. Notice that $j^{*}=1\left(j^{*}=L\right)$ corresponds to the side of dominant (non-dominant) spin domain, while $j=1(j=L)$ is the side of positive (negative) magnetic field.

Then, it is possible to evaluate the average relative growing interface $\left\langle I R_{j^{*}}\right\rangle$ by taking into account interface coordinates measured at different times between $t_{i}$ and $t_{f}$, and also by averaging all longitudinal $i$-fixed slices, i.e.

$$
\left\langle I R_{j^{*}}\right\rangle=\frac{1}{L} \frac{1}{\left(t_{f}-t_{i}+1\right)} \sum_{i=1}^{L} \sum_{t=t_{i}}^{t_{f}} I R_{i, j^{*}}(t) .
$$

Figure 7 shows $\left\langle I R_{j^{*}}\right\rangle$ versus $j^{*}$ for different values of the surface magnetic field $H$, for a fixed temperature $T=0.6$ and a fixed lattice size $L=32$. From the figure it follows that three qualitatively distinct growth regimes can clearly be distinguished. Indeed, it is observed that, while for small fields the system grows with convex curvature, on increasing the fields the growth process enters into a regime of non-defined curvature, since the dominant spin domain partially wets the confinement wall, while the non-dominant domain does not. But then, further increasing the fields, a point is reached where the non-dominant spin domain also (partially) wets the wall and the growing interface turns concave. This qualitative behaviour has been observed for all temperatures and lattice sizes within the range of interest of this work.

To explore this phenomenon quantitatively, the behaviour of the contact angles between the growing interface and the confinement walls (as functions of temperature and magnetic field) has to be studied thoroughly. Clearly, two different contact angles must be defined, namely $\theta_{D}$ for the angle corresponding to the dominant spin cluster, and $\theta_{N D}$ for the one that corresponds to the non-dominant spin cluster. Figure 8 shows plots of $\cot (\theta)$ versus $H$ for $T=0.9$ and $L=12$. The vertical dashed lines indicate the fields that separate a given growth regime from another one. One observes that, on increasing the surface fields, the growth regime changes from convex to non-defined to concave, in agreement with the interface profiles plotted in figure 7. Analogously, figures $9(a)-(d)$ show plots of $\cot (\theta)$ versus $T$ for $L=12$ and several different values for the magnetic field $H$. Again, vertical dashed lines correspond to temperatures of transitions between different growth regimes. Figure $9(a)$ corresponds to $H=$ 0.2 and displays the characteristic behaviour for very small magnetic fields- that is, a convex growing interface irrespective of temperature. For $H=0.4$ one observes a single transition from the growth regime of non-defined curvature to the convex growth regime, which shows up on increasing the temperature, as shown in figure $9(b)$. It should be noticed that the concave growth regime is prevented, since for small enough magnetic fields $\cot \left(\theta_{N D}\right)<0$ for all $T$. As 


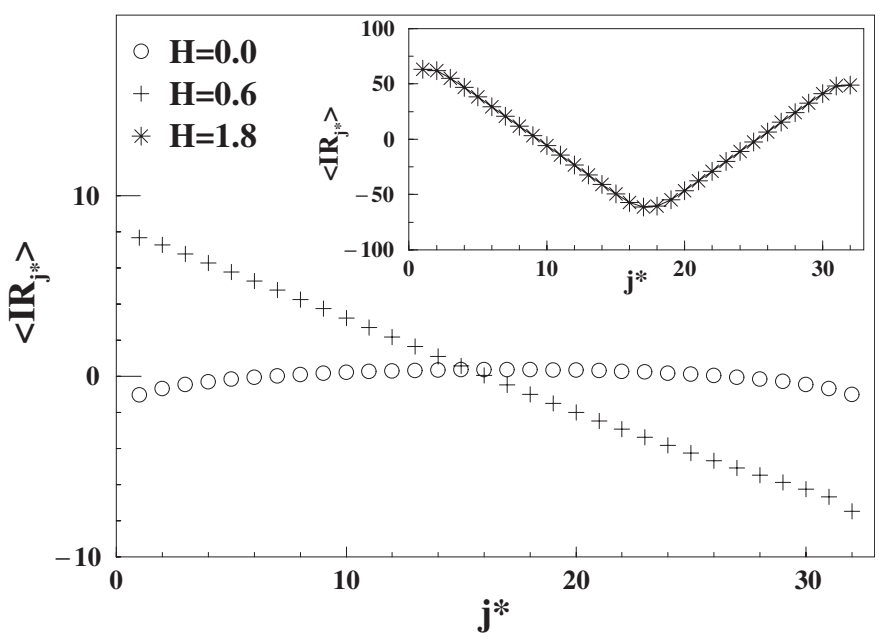

Figure 7. Plots of the averaged interface profile $\left\langle I R_{j^{*}}\right\rangle$ versus $j^{*}$ for $T=0.6$ and different values of the surface magnetic field $H$, as indicated. The lattice width is $L=32$. The plot corresponding to $H=1.8$ is shown separately in the inset, in order to allow a detailed observation of the profiles for lower $H$-values. The side $j^{*}=1\left(j^{*}=L\right)$ is the one corresponding to the dominant (nondominant) spin domain. On increasing the surface fields, the curvature of the growing interface changes: convex $\rightarrow$ non-defined $\rightarrow$ concave. This qualitative behaviour has been observed for all temperatures and lattice sizes within the range of interest of this work.

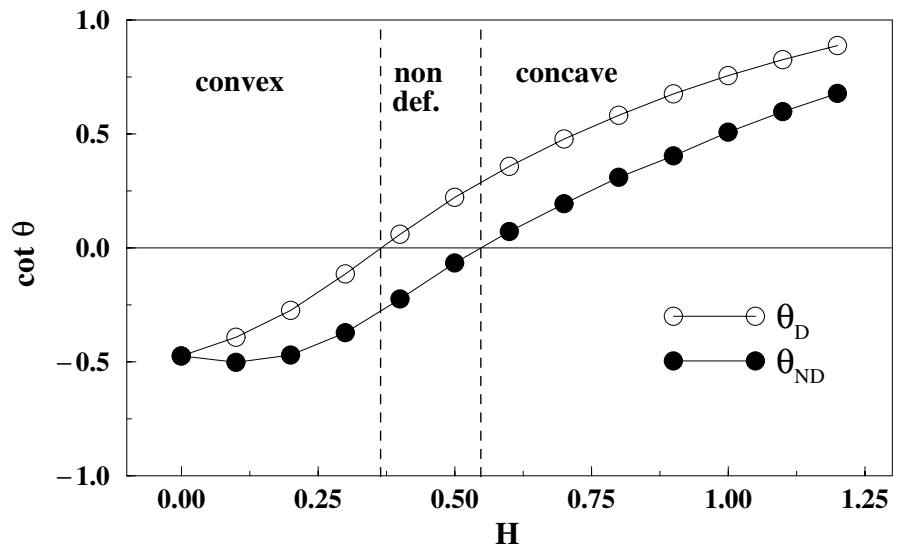

Figure 8. Plots of $\cot (\theta)$ versus $H$ for $T=0.9$ and $L=12 . \theta_{D}\left(\theta_{N D}\right)$ is the contact angle corresponding to the dominant (non-dominant) spin cluster, and is represented by open (filled) circles. The vertical dashed lines mark the fields that separate a given growth regime from another one, as indicated. A reference line corresponding to $\cot (\theta)=0$ has also been included.

the fields are increased, $\cot \left(\theta_{N D}\right)$ moves upwards and $\operatorname{crosses} \cot \left(\theta_{N D}\right)=0$, as expected from the plot of figure 8. For instance, the plots of $\cot (\theta)$ versus $T$ for $H=0.6$, shown in figure $9(c)$, exhibit this behaviour. Hence, here one has to deal with three transition temperatures. Finally, on further increasing the fields, the whole low-temperature region is dominated by the concave growth regime and two transition temperatures remain, as shown in figure $9(d)$ for $H=1.5$. All these features are compactly shown in the $H-T$ phase diagram of figure 3 , where open (filled) circles refer to the transition between non-defined and concave (convex) growth regimes. 

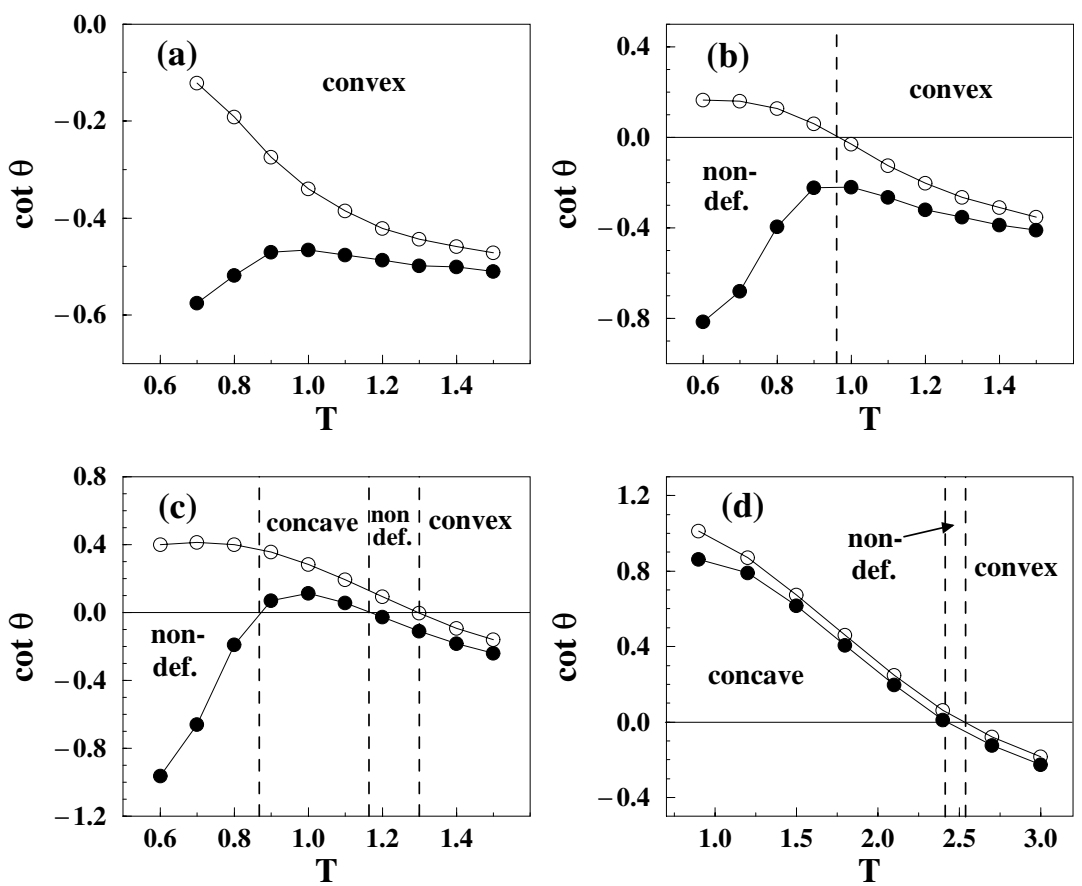

Figure 9. Plots of $\cot (\theta)$ versus $T$ for $L=12$ and several different magnetic fields: $(a) H=0.2$, (b) $H=0.4$, (c) $H=0.6$, and $(d) H=1.5 . \theta_{D}\left(\theta_{N D}\right)$ is the contact angle corresponding to the dominant (non-dominant) spin cluster, and is represented by open (filled) circles. The vertical dashed lines mark the temperatures that separate a given growth regime from another one, as indicated. Reference lines corresponding to $\cot (\theta)=0$ have also been included.

As shown in figure 3, the phase diagram of the MEM in a confined geometry with competing surface fields is very rich and exhibits eight regions. In order to gain some insight into the physics involved in this phase diagram, some typical snapshot configurations characteristic of the various different growth regimes observed are shown in figure 4 , as obtained using lattices of size $L=32$.

To begin with, let us analyse region I (see figure 3), that corresponds to the Ising-like non-wet state and the convex growth regime. In this region, temperature is low and the system grows in an ordered state, i.e. the dominant spin domain prevails and the deposited particles tend to have their spins all pointing in the same direction. Small clusters with the opposite orientation may appear preferably on the surface where the non-dominant orientation field is applied. These 'drops' might grow and drive a magnetization reversal, thus changing the sign of the dominant domain. In fact, the formations of sequences of well ordered domains are characteristic of the ordered phase of confined (finite-size) spin systems. For instance, this phenomenon has already been observed in finite Ising strips [23] and magnetic Eden thin films [45]. Due to the fact that open boundary conditions are imposed at $j=1$ and $L$, perimeter sites at the confinement walls experience a missing-neighbour effect; that is, the number of $\mathrm{NN}$ sites is lower than for the case of perimeter sites on the bulk. Since the surface magnetic fields in this region are too weak to compensate for this effect, the system grows preferentially along the centre of the sample as compared to the walls, and the resulting growth interface exhibits a convex shape. A typical snapshot configuration characteristic of region I is shown in figure $4(a)$.

Let us now consider an increase in the fields, such as the system may encounter in region 
II (see figure 3). Since the temperature is kept low, the system is still in its ordered phase and neighbouring spins grow preferentially parallel oriented. The surface fields in this region are stronger and thus capable of compensating for the missing NN sites on the surfaces. But, since the fields on both surfaces have opposite signs, it is found that, on the one hand, the field that has the same orientation as the dominant spin cluster favours the growth of surface spins, while on the other hand, the sites on the surface with opposite field have a lower probability of being chosen during the Monte Carlo growth process. Hence, the contact angle corresponding to the dominant spin cluster is then $\theta_{D}<\frac{\pi}{2}$, while the non-dominant one is $\theta_{N D}>\frac{\pi}{2}$. Thus, on the disfavoured side the growing interface becomes pinned and the curvature of the growing interface is not defined. Figure 4(b) shows a typical snapshot corresponding to region II.

Keeping $H$ fixed within region II but increasing the temperature, thermal noise will enable the formation of drops on the disfavoured side that eventually may nucleate into larger clusters as the temperature is increased even further. This process may lead to the emergence of an up-down interface, separating oppositely oriented domains, running in the longitudinal direction (i.e. parallel to the walls). Since sites along the up-down interface are surrounded by oppositely oriented NN spins, they have a low growing probability. So, in this case the system grows preferably along the confinement walls and the growing interface is concave (figure $4(c)$ ). Then, as the temperature is increased, the system crosses to region A (see figure 3) and the onset of two competitive growth regimes is observed, namely: (i) one exhibiting a non-defined growing curvature that appears when a dominant spin orientation is present, as in the case shown in figure 4(b); (ii) another that appears when an up-down interface is established and the system has a concave growth interface, as is shown in figure 4(c). On further increasing the temperature and for large enough fields, the formation of a stable longitudinal up-down interface that pushes back the growing interface is observed. So, the system adopts the concave growth regime (see figure $4(c)$ corresponding to region IV in figure 3 ). Increasing the temperature beyond $T_{c}(L)$, a transition from a low-temperature ordered state (region IV) to a high-temperature disordered state (region VI; see figure 4(e)), both within the concave growth regime, is observed. Analogously, for small enough fields, a temperature increase drives the system from the ordered convex growth regime (region I) to the disordered convex growth regime (region V; see figure $4(d)$ ). As shown in figure 3, there is also an intermediate fluctuating state (region $\mathrm{B}$ ) between regions $\mathrm{V}$ and VI, characterized by the competition between the disordered convex growth regime and the disordered concave one.

Finally, a quite unstable and small region (region III) that exhibits the interplay among the growth regimes of the contiguous regions can also be identified. Since the width of region III is of the order of the rounding observed in $T_{c}(L)$, large fluctuations between ordered and disordered states are observed, as well as from growth regimes of non-defined curvature to convex ones. However, figure $4(f)$ shows a snapshot configuration that is the fingerprint of region III, that may prevail in the thermodynamic limit-namely a well defined spin up-down interface with an almost flat growing interface.

Let us now extrapolate our results to show that the rich variety of phenomena found in a confined geometry are still present in the thermodynamic limit $(L \rightarrow \infty)$, leading to the phase diagram shown in the inset of figure 3 . As clearly seen by comparison with the finitesize results, the crossover regions $\mathrm{A}$ and $\mathrm{B}$ collapse in this limit, so only the six regions that correspond to well identified growth regimes (as illustrated by the snapshot configurations of figure 4) appear to remain.

In order to illustrate the extrapolation procedure, the following seven representative points of the finite-size phase diagram are discussed in detail: (i) the points labelled $P_{1}, P_{1}^{*}, P_{2}$, and $P_{2}^{*}$, that correspond to the intersections of the $H=0.6$ line with the various transition curves shown in figure 3 , and (ii) the points labelled $P_{3}, P_{3}^{*}$, and $P_{4}$, that refer to the intersection 




Figure 10. Plots of $T$ versus $L^{-1}$ for $12 \leqslant L \leqslant 48$, corresponding to the points $P_{1}, P_{1}^{*}, P_{2}$, and $P_{2}^{*}$, all of them with $H=0.6$. The fits to the data (solid lines) show that, within error bars, $P_{i} \rightarrow P_{i}^{*}(i=1,2)$ for $L \rightarrow \infty$.

points between regions I, II, III, and A, the minimum of the limiting curve between regions IV-VI and A-B, and the zero-field transition point, respectively.

Figure 10 shows plots of $T$ versus $L^{-1}$ for $12 \leqslant L \leqslant 48$ corresponding to the points $P_{1}$, $P_{1}^{*}, P_{2}$, and $P_{2}^{*}$. Also shown in the figure are the fits to the data extrapolated to $L^{-1}=0$. The results from the extrapolations are: $T_{1}=0.67 \pm 0.01, T_{1}^{*}=0.66 \pm 0.01$, and $T_{2}=1.30 \pm 0.02$, $T_{2}^{*}=1.29 \pm 0.01$, indicating that, within error bars, $P_{i} \rightarrow P_{i}^{*}(i=1,2)$ in the $L \rightarrow \infty$ limit. Using the same procedure, the extrapolations of $P_{3}$ and $P_{3}^{*}$ (not shown here) give: $H_{3}=$ $0.30 \pm 0.01, H_{3}^{*}=0.31 \pm 0.02$, and $T_{3}=0.69 \pm 0.01, T_{3}^{*}=0.71 \pm 0.03$. So, one has $P_{3} \rightarrow P_{3}^{*}$ for $L \rightarrow \infty$ within error bars. Finally, the extrapolation of $P_{4}$ is $T_{4}=T_{c}=0.69 \pm 0.01$.

Using the above-mentioned extrapolation procedure, the phase diagram in the thermodynamic limit can be drawn, as shown in the inset of figure 3. By comparison with the finite-size phase diagram of figure 3, one can note that, as anticipated, the crossover regions A and $\mathrm{B}$ appear in the phase diagram just as a consequence of the finite-size nature of confined geometries, since they collapse in the $L \rightarrow \infty$ limit. Moreover, we conjecture that region III may remain in the thermodynamic limit. Although this (very tiny!) region corresponds to a physically well characterized growth regime, since one expects that the system in this region may grow in an ordered phase with a delocalized up-down domain interface and a convex growing interface, statistical errors due to large fluctuations close to criticality hinder a more accurate location of this region. The unambiguous clarification of our conjecture remains as an open question that will require a huge computational effort.

Besides an Ising-like continuous wetting transition, coupled morphological transitions in the growing interface, which arise from the MEMs kinetic growth process, have also been identified. Comparing the equilibrium wetting phase diagram of the Ising model $[15,23,24]$ and that of the MEM, it follows that the non-equilibrium nature of the latter introduces new and rich physical features of interest: the non-wet (wet) Ising phase splits out into regions I and II (regions III and IV), both within the ordered regime $\left(T<T_{c}\right)$ but showing an additional 
transition in the interface growth mode. Also, the disordered state of the Ising system $\left(T>T_{c}\right)$ splits out into regions V and VI exhibiting a transition in the interface growth mode.

It should be noticed that we have restricted ourselves to temperatures above $T=0.5$ throughout, since the lower the temperature in the ordered phase, the greater the computational effort needed to reliably sample the whole configuration space (indeed, ergodicity is broken in the $T \rightarrow 0$ limit). Right at $T=0.5$ the wetting curve of the phase diagram (inset of figure 3 ) intercepts the $H$-axis close to $H=0.9$. On physical grounds, no particular features of interest are expected to arise in the $T \rightarrow 0$ limit, and the critical field $H=1$ for $T=0$ can be inferred by energetic considerations, as e.g. in the case of the Ising model.

\section{Conclusions}

The growth of magnetic Eden clusters with ferromagnetic interactions between NN spins has been studied in a $(2+1)$-dimensional geometry with competing surface magnetic fields. Extensive Monte Carlo simulations allow us to locate, on the one hand, an Isinglike localization-delocalization wetting transition, and, on the other hand, a morphological transition associated with the curvature of the growing interface. In this way, eight different regions on the $H-T$ phase diagram for a finite-size lattice are identified. Moreover, the characteristic behaviour of typical growth processes within each region are discussed, and qualitative explanations that account for the observed features are provided. Finally, extrapolating the results to the $L \rightarrow \infty$ limit the phase diagram is obtained. It is composed of six different regions, since two crossover regions identified in the finite-size phase diagram appear to collapse in the thermodynamic limit. The phase diagram obtained shows new and rich physical features of interest, which arise as a consequence of the non-equilibrium nature of the model investigated.

We hope that the results presented will, on the one hand, contribute to the understanding of the rich and complex physical phenomena exhibited by the irreversible growth of binary mixtures in confined geometries, and, on the other hand, stimulate further experimental and theoretical work.

\section{Acknowledgments}

This work was supported financially by CONICET, UNLP, and ANPCyT (Argentina). The authors thank the referees for fruitful comments and suggestions.

\section{References}

[1] de Gennes P G 1985 Rev. Mod. Phys. 57827

[2] Sullivan D E and Telo da Gama M M 1986 Fluid and Interfacial Phenomena ed C A Croxton (New York: Wiley)

[3] Dietrich S 1988 Phase Transitions and Critical Phenomena vol 12, ed C Domb and J L Lebowitz (New York: Academic) p 1

[4] Forgacs G, Lipowsky R and Nieuwenhuizen Th M 1991 Phase Transitions and Critical Phenomena vol 14, ed C Domb and J L Lebowitz (London: Academic)

[5] Parry A O 1996 J. Phys.: Condens. Matter 810761

[6] Steiner U, Eiser E, Klein J, Budkowski A and Fetters L J 1992 Science 2581126

[7] Budkowski A, Steiner U, Klein J and Schatz G 1992 Europhys. Lett. 18705

[8] Steiner U and Klein J 1996 Phys. Rev. Lett. 772526

[9] Taborek P and Rutledge J E 1992 Phys. Rev. Lett. 682184

[10] Ketola K S, Wang S and Hallock R B 1992 Phys. Rev. Lett. 68201

[11] Wyatt A F G, Klier J and Stefanyi P 1995 Phys. Rev. Lett. 741151

[12] Kozhevnikov V F, Arnold D I, Naurzakov S P and Fisher M E 1997 Phys. Rev. Lett. 781735 
[13] Hensel F and Yao M 1997 Eur. J. Solid State Inorg. Chem. 34861

[14] Christenson H K 1994 Phys. Rev. Lett. 73182

[15] Parry A O and Evans R 1990 Phys. Rev. Lett. 64439 Parry A O and Evans R 1992 Physica A 181250

[16] Swift M R, Owczarek A L and Indekeu J O 1991 Europhys. Lett. 14465

[17] Maciolek A and Stecki J 1996 Phys. Rev. B 541128

[18] Maciolek A 19961996 J. Phys. A: Math. Gen. 293837

[19] Carlon E and Drzewiński A 1998 Phys. Rev. E 572626

[20] Frisch H L, Puri S and Niebala P 1999 J. Chem. Phys. 11010514

[21] Liu H, Bhattacharya A and Chakrabarti A 1988 J. Chem. Phys. 1098607

[22] Mueller M, Albano E V and Binder K 2000 Phys. Rev. E 625281

[23] Albano E V, Binder K, Heermann D W and Paul W 1989 Surf. Sci. 223151

[24] Binder K, Landau D P and Ferrenberg A M 1995 Phys. Rev. Lett. 74298 Binder K, Landau D P and Ferrenberg A M 1995 Phys. Rev. E 512823

[25] Binder K, Landau D P and Ferrenberg A M 1995 Phys. Rev. E 535023

[26] Wener A, Schmid F, Mueller M and Binder K 1997 J. Chem. Phys. 1078175

[27] Ferrenberg A M, Landau D P and Binder K 1998 Phys. Rev. E 583353

[28] Hinrichsen H, Livi R, Mukamel D and Politi A 1997 Phys. Rev. Lett. 792710

[29] Eden M 1958 Symp. on Information Theory in Biology ed H P Yockey (New York: Pergamon) Eden M 1961 Proc. 4th Berkeley Symp. on Mathematics, Statistics and Probability vol 4, ed F Neyman (Berkeley, CA: University of California Press) p 223

[30] Family F and Vicsek T 1991 Dynamics of Fractal Surfaces (Singapore: World Scientific)

[31] Bunde A and Havlin S (ed) 1991 Fractals and Disordered Media (Heidelberg: Springer)

[32] Bunde A and Havlin S (ed) 1995 Fractals in Science (Heidelberg: Springer)

[33] Barabasi A L and Stanley H E 1995 Fractal Concepts in Surface Growth (New York: Cambridge University Press)

[34] Marsili M, Maritan A, Toigo F and Banavar J R 1996 Rev. Mod. Phys. 68963

[35] Albano E V, Binder K, Heermann D W and Paul W 1989 J. Chem. Phys. 913700

[36] Albano E V, Binder K, Heermann D W and Paul W 1990 J. Stat. Phys. 61161

[37] Binder K and Landau D P 1992 J. Chem. Phys. 961444

[38] Binder K, Niebala P and Pereyra V 1997 Z. Phys. B 10481

[39] Albano E V, Binder K and Paul W 1997 J. Phys. A: Math. Gen. 303285

[40] Díaz-Ortiz A, Sánchez J M and Morán-López J L 1998 Phys. Rev. Lett. 811146

[41] Albano E V, Binder K and Paul W 2000 J. Phys.: Condens. Matter 122701

[42] Ausloos M, Vandewalle N and Cloots R 1993 Europhys. Lett. 24629 Vandewalle N and Ausloos M 1994 Phys. Rev. E 50 R635

[43] Candia J and Albano E V 2000 Eur. Phys. J. B 16531

[44] Candia J and Albano E V 2001 Phys. Rev. E 63066127

[45] Candia J and Albano E V 2001 J. Appl. Phys. 905395

[46] Candia J and Albano E V 2002 Phys. Rev. Lett. 88016103

[47] Parry A O and Evans R 1991 Phys. Rev. Lett. 662175

[48] Indekeu J O, Owczarek A L and Swift M R 1991 Phys. Rev. Lett. 662174 